\title{
ADF-STEM imaging of c-Si/a-Si interfaces
}

\author{
Yanfa Yan, Qi Wang, Matt Page, Tihu Wang, and M.M. Al-Jassim \\ National Renewable Energy Laboratory, Golden, CO 80401
}

Surface passivation of c-Si is a critical process for many applications, especially heterojunction solar cells. The surface states often act as recombination centers for the charge carriers. The reduction of excess carrier loss at the c-Si surface, which is the part of heterojunction of an a-Si/c-Si solar cell, is one of the key issues to improve the device performances [1]. Hydrogenated amorphous silicon (a$\mathrm{Si}: \mathrm{H}$ ) has been demonstrated to be a promising candidate for surface passivation to c-Si surfaces [2]. The key issue for efficient passivation is the formation of high quality c-Si/a-Si interfaces. However, direct deposition of a-Si:H on a hydrophobic c-Si surface often leads to a mixed phase or epitaxial Si growth with some degree of structural defects, especially by hot-wire chemical vapor deposition (HWCVD), which prevents from achieving a better surface passivation and heterojunction formation. We have found that crystal surface treatments by atomic $\mathrm{H}$ and/or $\mathrm{NHx}$ radicals prior to the growth of thin hydrogenated amorphous silicon (a-Si:H) films can significantly improve the quality of c-Si/a-Si interfaces. In this work, we present an investigation of the quality of HWCVD grown c-Si/a-Si interfaces by high-resolution transmission electron microscopy (HRTEM), high-angle annular dark field scanning TEM imaging (ADF-STEM or Z-contrast), and electron energy loss spectroscopy (EELS).

The HR-TEM and ADF-STEM measurements were carried out using an FEI Tecnai TF20UT microscope. The EELS spectra were obtained using a Gatan GIF system.

Figure 1 left panel shows a HR-TEM image of a c-Si/a-Si interface. The growth of the a-Si layer is clearly demonstrated. The right panel of Fig. 1 shows an ADF-STEM image of the same c-Si/a-Si interface. Unlike the HR-TEM image, the ADF-STEM image reveals that the a-Si layer exhibits a two-sub-layer feature. It is seen that the a-Si layer with a thickness of about $2 \mathrm{~nm}$, as indicated by the two white dashed lines, shows higher intensity than the rest of the a-Si layers.

EELS spectra were used to find the difference between these two layers. Figure 2 shows the EELS spectra taken from three different areas, p1, p2, and p3. We found that the first $2 \mathrm{~nm}$ a-Si layer contains a large amount of $\mathrm{N}$, as seen in the left panel. This a-Si(N) layer could be the result of the NHx treatment. The right panel of Fig. 2 shows the fine structure of the Si-K edges obtained from the three different areas. Differences are clearly seen, indicating that the a-Si(N) layer may not act exactly the same as the pure a-Si layer.

\section{References}

[1] T. Wang, Q. Wang, M. Page, and T. Ciszek, Thin Solid Films, 430, 261 (2003).

[2] J. Yang and S. Guha, Mat. Res. Soc. Proc, 557, 239 (1999).

[3] This work was performed under DOE Contract \#DE-AC36-99GO10337. 

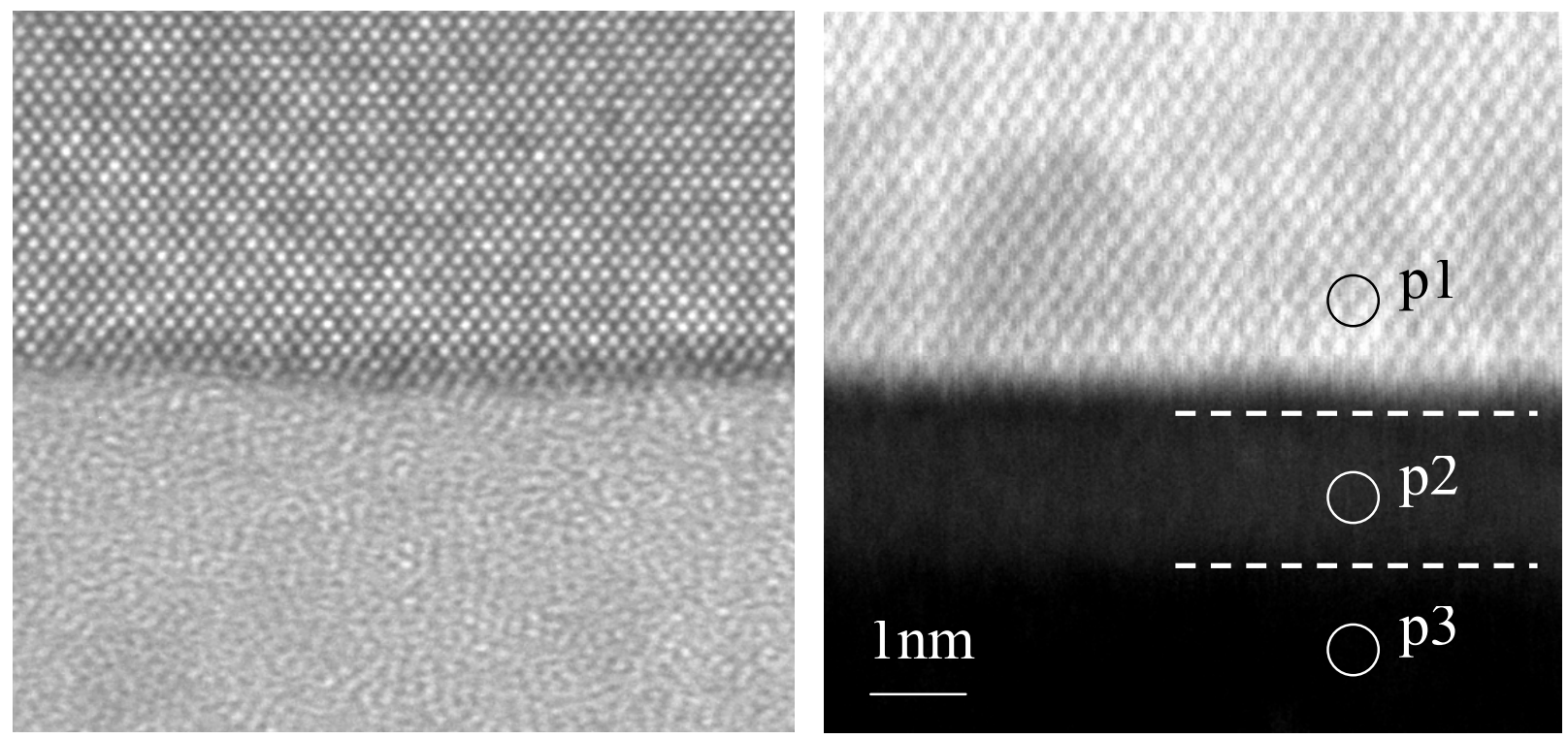

FIG. 1. (Left) HR-TEM image of a c-Si/a-Si interface. The achievement of high-quality a-Si layer is clearly seen. (Right) ADF-STEM image of the same c-Si/a-Si interface. It reveals that the a-Si layer consists of two sub-layers. The sub-layer indicated by the two white dashed-lines shows higher intensity than the rest of the a-Si layer.

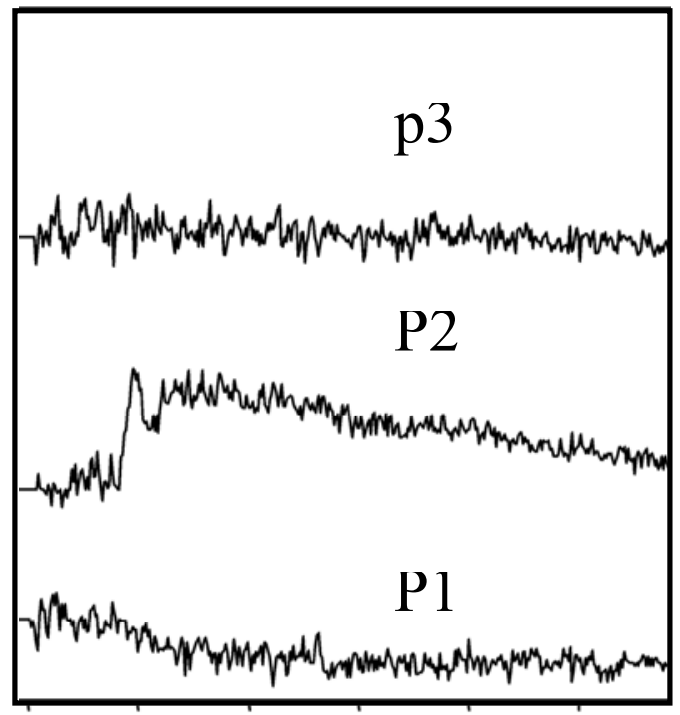

$350 \quad 400 \quad 450 \quad 500 \quad 550 \quad 600$

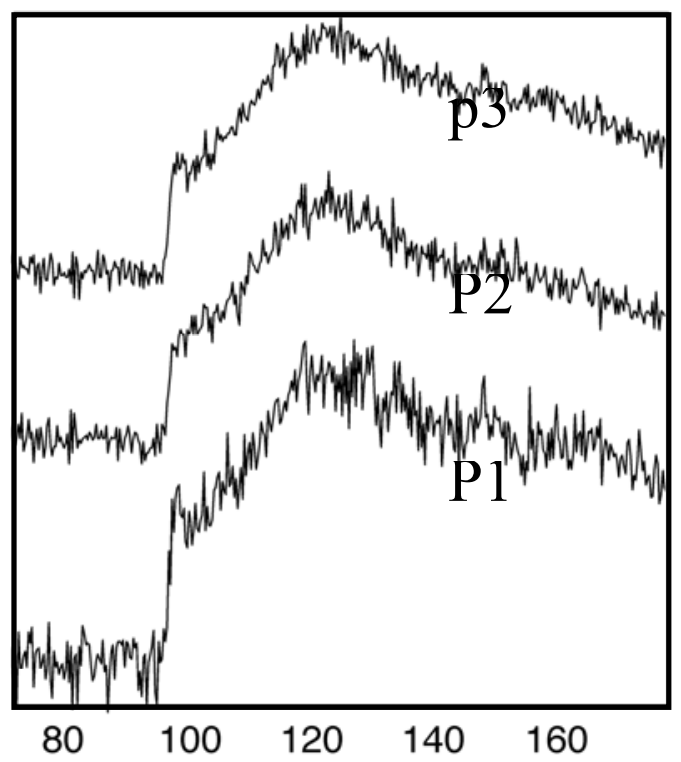

FIG. 2. EELS spectra obtained from three different areas, p1, p2, and p3, as shown in Fig. 1. The left panel shows the fine structure of N-K edges and the right panel shows the fine structure of Si-K edges. 